Marco Pioli

Universidad Complutense Madrid

https://doi.org/10.18778/8220-506-0.14

\title{
LA DIDATTICA DELLA LETTERATURA CON STUDENTI UNIVERSITARI DI ITALIANO COME LINGUA STRANIERA: UN PERCORSO SULLA "SICILIANITÀ” DI LEONARDO SCIASCIA
}

Riassunto: Conl'affermazione dei metodi comunicativi, a partire dagli anni Settanta del $\mathrm{XX}$ secolo la letteratura è stata progressivamente rilegata ai margini dell'insegnamento delle lingue straniere, per ritrovare una riabilitazione della sua opportunità solamente nel recente dibattito glottodidattico. Data questa premessa, il presente contributo sottopone una riflessione sulle peculiarità dell'educazione letteraria in lingua straniera in ambito universitario, un settore di indagine in realtà finora non troppo saggiato dagli studiosi e dal panorama editoriale specialistico. Al discorso teorico seguirà la presentazione di alcune strategie didattiche sperimentate all'interno di un percorso universitario dedicato all'ispanofilia di Leonardo Sciascia e al suo intrecciarsi con il peculiare modo con cui l'autore siciliano ha espresso la propria italianità mediante l'attaccamento all'isola natale.

Parole chiave: italiano LS, didattica della letteratura, studenti universitari, Leonardo Sciascia, Sicilia.

Abstract: The teaching of literature with university students of Italian as a foreign language: a path through Leonardo Sciascia's "Sicilianness". With the rise of communicative methods, since the 1970s literature has been gradually relegated to the margins of the teaching of foreign languages and only recently the pedagogical debate has reconsidered its potentialities. Given the previous premise, the present study proposes a reflection on the peculiarities of literary education in foreign language teaching at university, a research field which has been a bit neglected by scholars and specialized publishing houses so far. The references to theory will be followed by the presentation of some teaching strategies experimented in a university course dedicated to Leonardo Sciascia's Hispanophilia and to its intertwining with the peculiar way in which the Sicilian author expressed his Italianness through the attachment to his native island.

Keywords: Italian as foreign Language, Teaching of Literature, University Students, Leonardo Sciascia, Sicily. 


\section{La letteratura nella didattica dell'italiano LS}

A partire dagli anni Settanta del XX secolo, con l'affermazione dei metodi comunicativi, la letteratura è stata progressivamente rilegata ai margini dell'insegnamento delle lingue straniere ${ }^{1}$. L'azione dell'educazione linguistica si è indirizzata allo sviluppo della cosiddetta competenza comunicativa, ossia al dominio delle componenti strettamente linguistiche (fonetiche, ortografiche, morfosintattiche, lessicali e testuali) e degli aspetti extralinguistici e sociopragmatici di una lingua straniera (Balboni 2015: 34). Tipologie testuali più vicine alla norma parlata hanno soppiantato, perciò, la letteratura, la quale solamente negli ultimi decenni sta riemergendo dal "subconscio della glottodidattica" grazie alla progressiva affermazione dell'approccio ermeneutico (Magnani 2009: 113). Ispirato dalle tesi di Hans Hunfeld - docente di didattica della lingua inglese presso la Katholischen Universität EichstättIngolstadt -, tale approccio spinge gli studenti a interpretare attivamente il testo letterario in lingua straniera sulla base delle proprie esperienze pregresse e a confrontare reciprocamente i propri giudizi sotto la guida dell'insegnante. L'obiettivo è educare alla riflessione e alla complessità all'interno di esperienze comunicative reali.

Le tesi di Hunfeld, inoltre, risultano funzionali alla conciliazione di due campi d'azione didattica per lungo tempo rimasti separati tra loro: quello dell'educazione linguistica e quello dell'educazione letteraria. Se l'azione della prima è indirizzata, come anticipato, allo sviluppo della competenza comunicativa, un'educazione letteraria ispirata ai principi ermeneutici combina il lavoro sulla componente linguistica con l'apertura ai valori culturali, estetici e psicologici di un testo, con il fine di arricchire le capacità espressive e criticovalutative degli studenti, le loro nozioni storico-artistiche, la motivazione e la maturazione esistenziale degli stessi. Pertanto, la competenza linguistica diventa un prerequisito e allo stesso tempo un obiettivo dell'educazione letteraria, tanto che Fabio Caon da alcuni anni sta proponendo alla didattica della letteratura il concetto di "competenza comunicativa letteraria" (Caon e Spaliviero 2015: 26-45) 2 .

Infine, occorre tenere presente che l'insegnamento linguistico e letterario condividono una meta educativa a cui il testo letterario può contribuire sostanzialmente: la cosiddetta competenza interculturale. Lesperienza della lettura, come risaputo, apre a "nuovi mondi possibili" (Todorov 2008:

1 La dicitura "lingua straniera" verrà usata nel corso di questo studio come iperonimo, includendo anche il contesto della L2.

2 La prima formulazione del modello della "competenza comunicativa letteraria" risale al 2013 (Caon 2013: 271-300). 
16-17) e l'esplorazione dei testi nella "comunità ermeneutica" - come Romano Luperini (2002: 91-98) ha chiamato la nuova classe di letteratura - allena lo straniamento, il decentramento e il relativismo dei punti di vista, ossia quelle abilità relazionali indispensabili per non offrire licenze all'etnocentrismo e poter così comunicare con chi possieda un "software mentale" differente, cioè con chi provenga da culture o co-culture eterogenee (Serragiotto 2004: 48-50) ${ }^{3}$.

Alla luce di questo breve inquadramento, il dialogo tra la cultura italiana, siciliana nella fattispecie, e quella spagnola proposto dalla produzione letteraria di Leonardo Sciascia può offrire un'occasione proficua di intersezione tra le tre direttrici educative appena analizzate.

\section{Leonardo Sciascia: sicilianità e ispanofilia}

Nel 1979, Leonardo Sciascia - ormai affermatosi nel panorama letterario internazionale dopo i primi romanzi sulla mafia - nell'intervista rilasciata alla giornalista francese Marcelle Padovani rispose in questi termini alla domanda se fosse ancora lecito considerarlo uno "scrittore siciliano":

c'è stato un progressivo superamento dei miei orizzonti, e poco alla volta non mi sono più sentito siciliano, o meglio, non più solamente siciliano. Sono piuttosto uno scrittore italiano che conosce bene la realtà della Sicilia, e che continua a esser convinto che la Sicilia offre la rappresentazione di tanti problemi, di tante contraddizioni, non solo italiani ma anche europei, al punto da poter costituire la metafora del mondo odierno (Sciascia 1979: 78).

L'intervista, pubblicata sotto l'emblematico titolo La Sicilia come metafora, definisce il rapporto dialettico che Sciascia istaurò tra la sua isola e la penisola italiana per denunciare i soprusi e le ingiustizie perpetrati dalle varie manifestazioni del potere. Tale sensibilità civile, come veniva confessato alla stessa Padovani (Sciascia 1979: 85), deve essere ricondotta all'esperienza antifascista dell'autore, la quale nell'asfissiante Italia dell'autarchia mussoliniana trovò modo di maturare a seguito delle notizie provenienti dalla vicina Spagna, sconvolta dalla Guerra civile.

Nel 1936, infatti, Sciascia aveva 15 anni e, nonostante la retorica di regime indicasse Francisco Franco come alleato, la notizia della presa di posizione filorepubblicana di scrittori e attori americani da lui amati, come Ernest

3 La metafora del "software mentale" si è imposta negli studi sulla comunicazione interculturale e proviene da Geert Hofstede, psicologo sociale, e da uno dei suoi volumi più noti: Cultures and Organizations: Software of the Mind, London, McGrawHill, 1991. 
Hemingway o Charlie Chaplin, lo condussero pian piano verso posizioni nettamente antifasciste. Nelle Parrocchie di Regalpetra (1956) è possibile ritrovare la passione delle scelte ideologiche di quegli anni, in cui la Spagna servì da specchio per riflettersi e capirsi:

mi trovai dall'altra parte. Ora quei nomi delle città di Spagna mi si intridevano di passione. Avevo la Spagna nel cuore. Quei nomi - Bilbao Malaga Valencia; e poi Madrid, Madrid assediata - erano amore, ancor oggi li pronuncio come fiorissero in un ricordo di amore. E Lorca fucilato. E Hemingway che si trovava a Madrid. E gli italiani che nel nome di Garibaldi combattevano dalla parte di quelli che chiamavano rossi. [... ] A pensare oggi a quegli anni mi pare che mai più avrò nella mia vita sentimenti così intensi, così puri (Sciascia 1956: 43-44).

Insieme all'antifascismo, dunque, Sciascia scoprì anche la sua ispanofilia. Ben presto, iniziò a studiare castellano da autodidatta, avvalendosi di un manuale della Sonzogno, e a leggere classici come El Quijote, i saggisti spagnoli di inizio Novecento - Ortega y Gasset, Eugenio d'Ors, Americo Castro - e i poeti vittime delle persecuzioni di Franco (Sciascia 2000: 29 e 31-32) 4 .

Tuttavia, dietro alla passione spagnola di Sciascia, oltre ai motivi ideologici menzionati, interveniva una sollecitazione ancora più endemica. In Pirandello e la Sicilia, Sciascia affermava infatti nel 1961: "se la Spagna è, come qualcuno ha detto, più che una nazione un modo di essere, è un modo di essere anche la Sicilia; e il più vicino che si possa immaginare al modo di essere spagnolo" (Sciascia 1961: 1045).

La continuità Spagna-Sicilia rappresenta in effetti il nodo centrale del rapporto di Sciascia con la cultura iberica, continuità che l'autore, senza ricadere in unarbitraria interpretazione psicosociale, spiegava con una precisa ragione storica. Nel reportage "Qui un siciliano ritrova i viceré", pubblicato sul Corriere della Sera l'8 aprile 1983, si legge a proposito:

andare per la Spagna è, per un siciliano, un continuo insorgere della memoria storica, un continuo affiorare di legami, di corrispondenze, di "cristallizzazioni" $[\ldots]$ con qualcosa di simile $[\ldots]$ a una ritrovata fraternità. E dico ritrovata pensando allo splendido dominio degli arabi che Spagna e Sicilia ebbero comune e che ancora accende parole e fantasia ${ }^{5}$.

4 Per un inquadramento delle conoscenze letterarie spagnole di Sciascia si veda González de Sande (2009: 123-216).

5 Il reportage è riprodotto anche in Ore di Spagna (Sciascia 2000: 59-62). 
Quello appena letto non rappresenta, nell'ampia produzione sciasciana, un caso isolato di scrittura reportistica. Le memorie siciliane e le numerose letture spagnole ispirarono, dagli anni Cinquanta, i numerosi viaggi che Sciascia intraprese, fino agli anni Ottanta, in terra iberica con a fianco la moglie Maria Andronico. Da questi incontri nacquero numerosi resoconti su periodico, alcuni dei quali, nel 1988, furono raccolti in Ore di Spagna, volume che comprende anche le fotografie di Ferdinando Scianna, spesso in viaggio con Sciascia.

Diquestaproduzioneèinteressante segnalare chegià daisuoi primi campioni la prospettiva del traveller Sciascia - come direbbe Walter Benjamin - è quella di chi si sposta "nel tempo invece che nello spazio". L'immaginario trasmesso da questa produzione odeporica è, infatti, alimentato dai continui raffronti tra penisola iberica e contesto siciliano, paragoni spesso indirizzati all'indagine di comuni problemi sociali o esperienze storiche (come l'Inquisizione o la Guerra civile, a cui molti siciliani parteciparono come volontari), ma anche tesi a indagare questioni artistiche e linguistiche. Si tratta di un "movimento pendolare" - per usare la fortunata definizione proposta da Ricciarda Ricorda (2001: 199) - che concede poco spazio al folklore e che se, da una parte, rende queste pagine poco variegate nei tratti, dall'altra le preserva dalle indulgenze all'esotismo o dall'oleografia turistica in cui spesso si è scivolato, nella cultura europea, quando si è parlato di Spagna e di spagnoli ${ }^{7}$.

È importante segnalare che la ricerca del "gioco delle somiglianze"8 tra le due terre del Mediterraneo interessa non solo la scrittura di reportage, ma anche i "viaggi di carta" dedicati da Sciascia al tema della Guerra civil, come il racconto l'Antimonio, compreso nella seconda edizione de Gli zii di Sicilia (1960).

Proprio a questa produzione odeporica è dedicato il percorso didattico che segue, il quale, oltre a proporre un modello integrato tra obiettivi linguistici, letterari e interculturali, vuole offrire anche un'occasione per riflettere sulle prerogative educative degli studenti universitari di letteratura italiana all'estero, un settore di indagine in realtà non troppo saggiato dagli studiosi e dal panorama editoriale specialistico.

6 L'espressione è usata da Walter Benjamin in "Die Wiederkehr des Flaneurs", recensione scritta nel 1929 per il volume Spazieren in Berlin dell'amico Franz Hessel (Benjamin 1971: 101).

7 Per un approfondimento dei tratti caratterizzanti l'immaginario spagnolo di Leonardo Sciascia si veda Pioli (2019: 427-441).

8 L'espressione riprende il titolo di un elzeviro, raccolto nel 1983 in Cruciverba, in cui Sciascia definisce una consuetudine gnoseologica per lui tipica dei siciliani, cioè quella di ricercare costantemente analogie tra persone o cose (Sciascia 1983: 989-993). 


\section{Strategie didattiche per insegnare letteratura italiana a studenti universitari in contesto $\mathrm{LS}$}

Dopo un certo ritardo rispetto al mercato editoriale inglese, francese e spagnolo, anche in Italia si dispone oggigiorno di una discreta produzione di materiali che consentono l'uso della letteratura come risorsa per l'insegnamento della lingua e della cultura italiana a studenti stranieri. Si hanno opere della tradizione "facilitate" con ricchi apparati di note e glossari; letture linguisticamente "semplificate" - spesso accompagnate da eserciziari -; e svariate antologie.

Nell'impossibilità di un'analisi accurata di questi materiali, ci si limita a segnalare che le selezioni interessano soprattutto la prosa novecentesca. Nella maggior parte dei casi, inoltre, la letteratura viene coinvolta per esercitare esclusivamente lessico e grammatica, senza una seria apertura verso questioni letterarie o interculturali. Tuttavia, l'aspetto che si vuole maggiormente sottolineare riguarda i destinatari di questi lavori. Oltre all'esclusione dei livelli di competenza pre-intermedi, la tendenza è quella di indirizzarsi a un profilo neutro di apprendente, forse identificabile con gli utenti degli Istituti italiani di cultura. Tale tendenza è riscontrabile anche nei lavori più strettamente compromessi con l'educazione letteraria, come il recente Letteratura italiana per stranieri di Paolo Balboni, pubblicato nel 2019 presso Edilingua, in cui non solo non si specifica un profilo target di apprendente, ma l'organizzazione dei contenuti non sempre aderisce alle necessità formative di chi ricerchi un equilibrio tra accessibilità all'input e rigore scientifico, come nel caso degli studenti universitari.

Il percorso che si presenta, dunque, cerca di proporre alcune strategie didattiche per intercettare le necessità appena segnalate.

Esso è stato ideato per gli studenti della laurea in Lingue e letterature straniere dell'Universidad Complutense di Madrid, nello specifico per gli iscritti al corso "La literatura italiana: interrelaciones y proyección", a cui generalmente si accede al terzo o al quarto anno accademico, dunque con un livello di competenza comunicativa pari al B2 del QCER (Consiglio d'Europa: 2002).

I contenuti sono stati organizzati in una lunga unità didattica di circa 12 ore metaforicamente intitolata "Leonardo Sciascia: viaggi e altri viaggi", e suddivisa in tre unità di acquisizione. La prima, "Leonardo Sciascia", restituisce un sintetico quadro bio-bibliografico dell'autore; la seconda, "Viaggi: analisi di un reportage", analizza la scrittura di reportage e l'immaginario siculo-spagnolo di Sciascia in relazione con gli stereotipi ricorrenti sulla penisola iberica; la terza, "Altri viaggi: introduzione di un racconto", infine, presenta L'antimonio, ricercando le analogie tra i viaggi reali e quelli immaginari di Sciascia. 
Inoltre, l'unità didattica intende approfondire i seguenti obiettivi linguistici e interculturali: 1) potenziare lessico e gli usi del passato remoto; 2) stimolare produzione orale e scritta in italiano; 3 ) riflettere sulle immagini culturali e sulla loro formazione; 4) esercitare le abilità relazionali e sviluppare competenza interculturale.

Dal punto di vista metodologico le indicazioni sull'esplorazione ermeneutica dei testi sono state combinate con i principi della pedagogia umanistico-affettiva. Il sostegno della motivazione degli apprendenti, perciò, è stata una priorità nella progettazione dell' intero percorso, il quale, comunque, affronta tematiche - quelle attinenti al viaggio - sicuramente vicine agli interessi e all'esperienza degli studenti.

Nell'esplorazione degli input sono stati seguiti i principi della percezione gestaltica della globalità, dell'analisi e della sintesi (Balboni 2015: 153). Gli studenti sono stati costantemente chiamati a generare ipotesi e a condividere i propri pareri interpretativi, in modo da stimolare attività ermeneutica e produzione linguistica. Per sostenere questi processi, il percorso prevede una diffusa gamefication e la possibilità di sviluppare quasi tutte le attività in coppia o in gruppi. All'insegnante aspetta il ruolo del regista, supportato da un Power point ricco di immagini e da un quaderno dello studente, di circa 20 pagine, composto dalle attività, dai testi da leggere, da un foglio di lavoro per gli esercizi e da una bibliografia finale.

Per esemplificare il lavoro svolto, si procederà a illustrare alcuni momenti della seconda unità di apprendimento, incentrata sulla lettura e l'analisi del primo reportage spagnolo di Sciascia, apparso su L'Ora di Palermo il 20 agosto 1966, intitolato "Com'era la Spagna" e dedicato alla città di Barcellona e al ricordo della Guerra civile.

Innanzitutto, si inizia generando ipotesi sul contenuto del reportage a partire dal suo incipit, "Barcellona, Albergo Colon” (Sciascia 1991: 168), mentre nella consegna dell'attività gli studenti possono ritrovare una precisa contestualizzazione del brano, con i relativi riferimenti bibliografici in nota, in modo da poter verificare le informazioni:

\section{MOTIVAZIONE.}

1. Proporre dei bilanci all'interno della produzione sciasciana è sicuramente operazione rischiosa, vista la smisurata quantità degli scritti dell'autore. Tuttavia, alla luce dei materiali recensiti dai repertori bibliografici in circolazione, ${ }^{8}$ il suo primo reportage d'argomento spagnolo può essere considerato quello pubblicato su L'Ora di Palermo il 20 agosto 1966, nella

9 Il testo, insieme ad altre collaborazioni di Sciascia su L'Ora di Palermo, è stato ripubblicato in Quaderno (Sciascia 1991: 168-175). 
rubrica Quaderno, diretta dall'autore tra il 1964 e il 1968, i cui testi sono stati raccolti in volume nel $1991^{9}$. Qui di seguito trovi l'incipit del reportage: cosa ti aspetti di trovarvi?

(8) Cfr. La memoria di carta: bibliografia delle opere di Leonardo Sciascia, (a c. di) Valentina Fascia, Milano, Otto/Novecento, 1998; A. Motta, Bibliografia degli scritti di Leonardo Sciascia, Palermo, Sellerio, 2009.

(9) Leonardo Sciascia, Quaderno, introduzione di Vincenzo Consolo, nota di Mario Farinella, Palermo, Nuova Editrice meridionale, 1991. Il reportage in analisi è alle pagine $168-175^{10}$.

Le ipotesi vengono appurate introducendo il proseguimento dell'incipit: "nel trentesimo anniversario dell'alzamiento" (Sciascia 1991: 168). Dalla parola "alzamiento", con un brainstormig, si ricavano dunque le informazioni storiche utili per avvicinare la lettura del reportage, la quale può essere silenziosa o ad alta voce, eseguita a casa o a lezione, in base alle tempistiche.

Il reportage, riprodotto nel quaderno dello studente, come allegato, è presentato integralmente, con in coda gli estremi dell'edizione di riferimento e delle pagine trascritte ${ }^{11}$. Laspetto che indirizza nettamente questa didattizzazione in direzione del target selezionato è il ricco lavoro di annotazione eseguito sul brano. Esso è servito per avvicinare linguisticamente il testo all'interlingua degli studenti, compito per il quale si è fatto ricorso, oltre che ai vari sillabi di livello B2, ai lemmi presenti nel Nuovo vocabolario di base della lingua italiana di Tullio De Mauro (2016) ${ }^{12}$. In questa operazione, la sinonimia è stata la strategia più usata, ma si è ricorso anche alle definizioni tramite gli iperonimi ("boccale: bicchiere alto e largo con manico") o alla parafrasi esplicativa dei termini sciasciani: in "aspettano i rinforzi che spazzeranno via la canaglia male armata" (Sciascia 1991: 168), il termine "canaglia" viene sciolto con: "insieme di persone malvage, disoneste, spregevoli".

Tuttavia, trattandosi di studenti universitari stranieri, è stato necessario chiarire anche itanti riferimenti storici, politici eletteraripropri dell'enciclopedia del mondo sciasciana. Dato che spesso a lezione sono presenti studenti provenienti da tradizioni culturali molto lontane, come i cinesi, sono state esplicitate anche informazioni che sarebbero scontate per un occidentale, come

10 Di qui in avanti si riproducono, insieme alle consegne, anche le note presenti nel quaderno dello studente, mantenendo la stessa numerazione.

11 Nel quaderno è presente la seguente dicitura: "Da Leonardo Sciascia, Quaderno, Palermo, Nuova Editrice meridionale, 1991, pp. 168-175”.

12 T. De Mauro, Il nuovo vocabolario di base della lingua italiana, 2016. Online: www.internazionale.it/opinione/tullio-de-mauro/2016/12/23/il-nuovovocabolario-di-base-della-lingua-italiana [25/01/2020]. 
il riferimento a Cristoforo Colombo (Sciascia 1991: 169) ${ }^{13}$. La stessa ottica transnazionale ha permesso di sciogliere gli impliciti culturali, categoria in cui vanno incluse anche le espressioni idiomatiche. Infine, per evitare il pericolo di bias, ossia di innescare crisi interculturali, si è proceduto con il massimo rigore storico nell'annotare i riferimenti ai protagonisti della Guerra civile ${ }^{14}$.

Tornando allo sviluppo delle attività, la comprensione viene verificata prima globalmente e poi con domande analitiche. Rispetto alla prima modalità, si segnala l'attività 4, in cui si proiettano quattro scatti di Ferdinando Scianna, tratti da Ore di Spagna, e si chiede agli studenti di scegliere quali userebbero per illustrare il testo, ovviamente giustificando la risposta ${ }^{15}$.

Successivamente si aprono tre sequenze di analisi, una linguistica e le altre due in linea con gli obiettivi letterari del percorso.

Della prima si segnala il crucipuzzle inserito nell'attività 7 , in cui si chiede agli studenti di rintracciare 15 parole estratte dalle glosse lessicali apposte al reportage e poi di abbinarle con i sinonimi usati da Sciascia. L'obiettivo è quello di portare gli studenti a interagire attivamente con il ricco apparato di annotazioni dell'allegato e di interiorizzarle grazie a una dinamica ludica.

La seconda sequenza analizza il ruolo che la Guerra civile ebbe nella scoperta spagnola di Sciascia, mentre la terza mette a confronto, in una prospettiva imagologica, la "sicilianità" e "l'ispanofilia" dell'autore. L'attività 18 presenta la nozione del "movimento pendolare" usata dalla Ricorda per definire le pagine di viaggio sciasciane, sempre con il rispettivo riferimento bibliografico:

18. Ricciarda Ricorda, esperta di letteratura di viaggio, sostiene che nelle pagine sciasciane ci sia un frequente "movimento pendolare". Pensa alla metafora del pendolo e al suo movimento. A cosa si riferisce la studiosa?

13 "Cristoforo Colombo (Genova, 1451 - Valladolid, 1506), navigatore genovese al servizio dei re cattolici spagnoli; scoprì il continente americano nel 1492".

14 Il riferimento al generale Franco (Sciascia 1991: 169) viene così sciolto: "Francisco Franco (Ferrol, 1892 - Madrid, 1975), militare e dittatore spagnolo che rimase al potere dalla vittoria nella Guerra civile spagnola alla sua morte, nel 1975. Nel luglio $1936 \mathrm{fu}$ tra i protagonisti, insieme ad altri generali, del colpo di stato militare contro la Seconda Repubblica spagnola”.

15 Le fotografie sono tratte dall'ultima edizione di Ore di Spagna per i tipi di Contrasto (Sciascia: 2016), in cui Scianna ha perfezionato il suo apparato fotografico rispetto al precedente volume Bompiani. Le foto ritraggono: un torero a Siviglia, 1983 (p. 21), il generale Enrique Líster, eroe repubblicano, Madrid, 1985 (p. 31), Belchite, 1984 (pp. 24-25), e il ballo della Sardana, Barcellona, 1984 (p. 104). 
(16) R. Ricorda, "Landare per la Spagna di un siciliano: immagini di viaggio", [in] Avevo la Spagna nel cuore, (a c. di) Natale Tedesco, Milano, La Vita Felice, 2001, p. 199.

Una volta che gli studenti abbiano riflettuto e commentato la ragione di tale definizione critica, si procede alla compilazione di una tabella, presente nel foglio di lavoro, utile a ricostruire i tanti paragoni presenti nel reportage tra la Spagna, la Sicilia e l'Italia.

La fase di sintesi intende fissare gli argomenti trattati attraverso la riflessione esplicita. L'attività 22 sottopone una sorta di gioco di ruolo letterario a partire da due citazioni, una di Walter Benjamin e una di Giacomo Debenedetti ${ }^{16}$, e si chiede agli studenti di dividersi in gruppi. Ogni gruppo decide di assumere l'identità dell'uno o dell'altro critico, elenca le ragioni della proprio scelta e tramite un portavoce prova a convincere il resto della classe della propria pertinenza nell'analisi delle pagine reportistiche di Sciascia. I gruppi assegneranno un voto a ogni portavoce in modo da poter decretare, alla fine, il "critico" più persuasivo. Lattività successiva sottopone, invece, la famosa citazione sciasciana sul comune "modo di essere" tra Sicilia e Spagna (Sciascia 1961: 1045) e spinge gli studenti, sulla base della propria esperienza, a confrontare l'immaginario dell'autore con la propria autoimmagine della Spagna e della Sicilia (isola, con molta probabilità, già avvicinata durante i corsi di lingua o attraverso i film, la letteratura, o magari anche direttamente, a seguito di un viaggio).

Infine, partendo da tre fotografie di Scianna sulla Semana santa andalusa ${ }^{17}$, gli studenti devono scrivere, individualmente, un breve reportage di circa 200 parole per accompagnare le tre immagini, cercando di assumere un punto di vista non stereotipato, ma personale, così come ha fatto Sciascia nel suo resoconto odeporico. Nella sperimentazione eseguita presso la Complutense, i testi sono stati condivisi su un apposito Padlet, in modo che ogni studente potesse confrontarsi con le proposte altrui, e alla fine, per stimolare ancora il gioco dei punti di vista, è stato condiviso il reportage sulla Semana santa scritto da Sciascia e contenuto in Ore di Spagna (2000: 47-49).

16 La citazione di Benjamin è quella menzionata in precedenza (1971: 101), mentre quella di Debenedetti (1971: 327-328) verte sulla necessità, nell'era della riproducibilità tecnica del viaggio, di forme più originali nella rappresentazione dell'esperienza dello spostamento. Di entrambe, lo studente ritrova nel quaderno le precise indicazioni bibliografiche.

17 Gli scatti ritraggono dei figuranti incappucciati, degli adolescenti e una signora immortalati a Granada e a Carmona (Sciascia 2016: 76, 151 e 81). 


\section{Considerazioni finali}

Oltre all'esercitazione creativa, per verificare la riuscita delle strategie didattiche impiegate è stato sottoposto anche un test di valutazione finale, del quale si presentano alcuni quesiti per avvicinare la conclusione.

Alla domanda 4, "Pensi che la letteratura possa essere un buono strumento per l'apprendimento della lingua italiana", il 93\% dei 28 studenti intervistati risponde con "molto" o "moltissimo". Le risposte alla domanda 7 rivelano che in due casi si preferisce la lezione frontale, in quattro la didattica attiva, mentre il resto del gruppo opta per un equilibrio tra le due modalità di insegnamento menzionate. I risultati del quesito 9, "Metti in ordine per importanza queste modalità di insegnamento letterario", dimostrano che le tecniche predilette sono le annotazioni e l'uso degli audiovisivi a discapito della didattica ludica. Se si relazionano questi risultati con quelli del quesito 12, "Pensi che sia importante che un materiale didattico di letteratura in lingua straniera per studenti universitari presenti precisi riferimenti bibliografici”, a cui il $93 \%$ risponde affermativamente, si capisce l'opportunità di pensare anche a questo profilo di apprendenti nella progettazione di materiali didattici letterari in italiano. Positivi poi sono i giudizi sull'interesse suscitato dalla scrittura di Leonardo Sciascia e dalla sua rappresentazione della Sicilia e della Spagna (solo in due casi non si è certi se si voglia continuare a leggere le pagine del siciliano).

Infine, per congedare non solo questa sperimentazione ma anche il presente studio, può valere la spontaneità di questo commento libero: "vorrei che insegnassero di più all'università in questo modo perché attira l'attenzione di più studenti".

\section{Bibliografia}

Balboni, Paolo Ernesto (2015). Le sfide di Babele. Insegnare le lingue nelle società complesse, Torino, Utet.

Balboni, Paolo Ernesto (2019). Letteratura italiana per stranieri, Roma, Edilingua.

Benjamin, Walter (1971). Immagini di città, postfazione di Peter Szondi, Torino, Einaudi.

Caon, Fabio (2013). "Dall'analisi testuale alla competenza comunicativa nella fruizione di testi letterari”, EL.LE, II (2), pp. 271-300.

Caon, Fabio e Spaliviero, Camilla (2015). Educazione letteraria, linguistica, interculturale: intersezioni, Torino, Loescher editore.

Consiglio d'Europa (2002). Quadro comune europeo di riferimento per le lingue: apprendimento, insegnamento, valutazione, Oxford, La Nuova Italia.

De Mauro, Tullio (2016). Il nuovo vocabolario di base della lingua italiana. Online: www. internazionale.it/opinione/tullio-de-mauro/2016/12/23/il-nuovo-vocabolario-dibase-della-lingua-italiana [25/01/2020].

Debenedetti, Giacomo (1971). Il romanzo del Novecento: quaderni inediti, Milano, Garzanti.

González de Sande, Estela (2009). Leonardo Sciascia e la cultura spagnola, introduzione di Sara Zappulla Muscarà, foto di Giuseppe Leone, Catania, La Cantinella. 
Hofstede, Geert (1991). Cultures and Organizations: Software of the Mind, London, McGrawHill.

Luperini, Romano (2002). Insegnare la letteratura oggi, Lecce, Manni.

Magnani, Mirco (2009). "Il testo letterario e l'insegnamento delle lingue straniere", Studi di Glottodidattica, 1, pp. 107-113.

Pioli, Marco (2019). "L'immaginario spagnolo di Leonardo Sciascia: genealogie mediterranee”, Italian Studies, 74 (4), pp. 427-441.

Ricorda, Ricciarda (2001). "Landare per la Spagna di un siciliano: immagini di viaggio", [in] Natale Tedesco (a c. di), Avevo la Spagna nel cuore, Milano, La Vita Felice, pp. 153-178.

Sciascia, Leonardo (1956). Le parrocchie di Regalpetra, [in] Leonardo Sciascia (2004), Opere 1956-1971, a c. di Claude Ambroise, Milano, Bompiani, pp. 1-170.

Sciascia, Leonardo (1961). Pirandello e la Sicilia, [in] Leonardo Sciascia (2004), Opere 19841989, a c. di Claude Ambroise, Milano, Bompiani, pp. 1041-1203.

Sciascia, Leonardo (1979). La Sicilia come metafora, intervista di Marcelle Padovani, Milano, Mondadori.

Sciascia, Leonardo (1983). Cruciverba, [in] Leonardo Sciascia (2004), Opere 1971-1983, a c. di Claude Ambroise, Milano, Bompiani, pp. 965-1282.

Sciascia, Leonardo (1991). Quaderno, introduzione di Vincenzo Consolo, nota di Mario Farinella, Palermo, Nuova Editrice meridionale.

Sciascia, Leonardo (2000). Ore di Spagna, introduzione di Natale Tedesco e fotografie di Ferdinando Scianna, Milano, Bompiani.

Sciascia, Leonardo (2016). Ore di Spagna, fotografie di Ferdinando Scianna, Roma, Contrasto. Serragiotto, Graziano (a c. di) (2004), CEDILS. Certificazione in didattica dell' italiano a stranieri, Roma, Bonacci.

Todorov, Tzvetan (2008). La letteratura in pericolo, Milano, Garzanti. 